\title{
Interplay of projectile breakup and target excitation in reactions induced by weakly bound nuclei
}

\author{
M. Gómez-Ramos* and A. M. Moro ${ }^{\dagger}$ \\ Departamento de FAMN, Facultad de Física, Universidad de Sevilla, Apdo. 1065, E-41080 Sevilla, Spain
}

(Received 31 August 2016; revised manuscript received 30 January 2017; published 16 March 2017)

\begin{abstract}
Background: Reactions involving weakly bound nuclei require formalisms able to deal with continuum states. The majority of these formalisms struggle to treat collective excitations of the systems involved. For continuumdiscretized coupled channels (CDCC), extensions to include target excitation have been developed but have only been applied to a small number of cases.

Purpose: In this work, we reexamine the extension of the CDCC formalism to include target excitation and apply it to a variety of reactions to study the effect of breakup on inelastic cross sections.

Methods: We use a transformed oscillator basis to discretize the continuum of the projectiles in the different reactions and use the extended CDCC method developed in this work to solve the resulting coupled differential equations. A new code has been developed to perform the calculations.

Results: Reactions ${ }^{58} \mathrm{Ni}(d, d){ }^{58} \mathrm{Ni}^{*},{ }^{24} \mathrm{Mg}(d, d){ }^{24} \mathrm{Mg}^{*},{ }^{144} \mathrm{Sm}\left({ }^{6} \mathrm{Li},{ }^{6} \mathrm{Li}\right){ }^{144} \mathrm{Sm}^{*}$, and ${ }^{9} \mathrm{Be}\left({ }^{6} \mathrm{Li},{ }^{6} \mathrm{Li}\right){ }^{9} \mathrm{Be}$ are studied. Satisfactory agreement is found between experimental data and extended CDCC calculations.

Conclusions: The studied CDCC method has proven to be an accurate tool to describe target excitation in reactions with weakly bound nuclei. Moderate effects of breakup on inelastic observables are found for the reactions studied. Cross-section magnitudes are not modified much, but angular distributions present smoothing when opposed to calculations without breakup.
\end{abstract}

DOI: 10.1103/PhysRevC.95.034609

\section{INTRODUCTION}

Few-body models have been very successful in describing nuclear reactions involving weakly bound nuclei, where breakup probabilities are high and continuum states of these nuclei influence heavily other relevant reaction channels such as elastic scattering. Many models able to deal with these positive-energy states have been developed, such as continuum-discretized coupled channels (CDCC) [1,2], the adiabatic approximation $[3,4]$, Faddeev-AGS equations [5,6], and several semiclassical approximations [7-12].

In most of these models, in particular CDCC, the weakly bound nucleus is considered to be composed of two subsystems, the valence particle and the core, which may be dissociated during the reaction in a breakup process. These subsystems are usually considered to be inert at the energies of interest. This is a good approximation for reactions involving deuterons, which were the origin of many of these models, but it is more questionable for more complex systems. For this reason, extensions to include the collective excitations of the core subsystem have been developed both for CDCC $[13,14]$ and Faddeev-AGS equations [15]. These extensions were able to give proper descriptions of reactions involving weakly bound nuclei with deformed cores such as ${ }^{11} \mathrm{Be}[16]$ and ${ }^{19} \mathrm{C}$ [17].

In general, these models also consider that the target is an inert system without internal degrees of freedom relevant for the reaction. Possible excitations of the target are assumed to be effectively included in the fragment-target optical potentials, rather than explicitly treated. This assumption makes these models unsuitable to describe excitation of the target or more

\footnotetext{
*mgomez40@us.es

†moro@us.es
}

generally any process in which both breakup of one system and collective excitation of the other may take place concurrently.

However, there exist a variety of measurements in which weakly bound nuclei collide with nuclei with collective degrees of freedom which are excited during the reaction, either due to a big deformation and an associated rotational spectrum or to the existence of low-energy vibrational levels $[18,19]$. These experiments require a consistent description of both breakup and collective excitation of the target if reliable information is to be obtained from them.

Extensions to include target excitation in CDCC were already developed in the 1980s by the Kyushu-Pittsburg groups [20] and have received some recent attention [21] but in general have been restricted to deuteron scattering considering only its $s$-wave component. Therefore, we find it timely to reexamine the corresponding formalism and apply it to more general reactions using the full formalism without introducing further approximations. In this respect, it must be remarked that the extension of the Faddeev-AGS equations which allowed for the inclusion of collective excitations of the core subsystem also permits the inclusion of target excitation thanks to the symmetric treatment of target and projectile which is employed in the Faddeev equations [15]. In this work we reexamine the extension of the CDCC method to include target excitation. The outline of this work is the following: in Sec. II the formalism used for the extension is presented, while calculations for different low- to medium-energy reactions are shown in Sec. III. Finally, the summary and conclusions are presented in Sec. IV.

\section{SCATTERING FRAMEWORK}

In this section we derive the expression for the coupling potentials which allow us to treat breakup of the projectile 
and excitation of the collective states of the target on an equal footing. Although these potentials were already derived by the Kyushu-Pittsburg group [20], we find it suitable to present them again here, in order to make this work more self-consistent noting that we use the $j-j$ coupling scheme, in contrast to [20], where they employed the $L-S$ coupling scheme.

In our framework, the projectile is modelled as a two-body system composed of a core $c$ and a valence particle $v, \vec{r}$ being their relative coordinate. The target is assumed to have some internal degrees of freedom $\xi$, which may be excited due to its interaction with the projectile. The effective Hamiltonian of the projectile-target system is of the form

$$
H(\vec{R}, \vec{r}, \xi)=T(\vec{R})+h_{p}(\vec{r})+h_{t}(\xi)+V_{p t}(\vec{r}, \vec{R}, \xi),
$$

where $\vec{R}$ is the relative coordinate between projectile and target, $T$ is the kinetic-energy operator of the projectile-target system, and $h_{p}$ is the internal Hamiltonian of the projectile:

$$
h_{p}(\vec{r})=T(\vec{r})+V_{v c}(\vec{r}),
$$

which depends on the relative coordinate between valence and core systems. We must remark that, in this formalism, $V_{v c}$ may depend on other internal coordinates of core or valence particle, resulting in eigenstates of the projectile which are a linear combination of single-particle components. However, it is assumed here that these internal coordinates will not be modified by the interaction with the core, so their influence is reduced to the structure of the projectile and will not affect the dynamics of the reaction. Thus, they are ignored in our derivation. Nevertheless, we note that the following derivation is fully applicable to multicomponent projectile wave functions, but components with different states of the core will not be coupled in this formalism.

In Eq. (1), $h_{t}$ is the internal Hamiltonian of the target, which only depends on collective degrees of freedom, whose identities will depend on the model chosen to describe the target. Finally, the interaction potential $V_{p t}$ is divided in the interaction between the valence particle and the target $V_{v t}$, and between the core and the target $V_{c t}$. Both interactions depend on the relative coordinate between each subsystem and the target and the internal degrees of freedom of the target; namely,

$$
\begin{aligned}
V_{p t}(\vec{r}, \vec{R}, \xi) & =V_{v t}\left(\vec{r}_{v}, \xi\right)+V_{c t}\left(\vec{r}_{c}, \xi\right), \\
\vec{r}_{v} & =\vec{R}-\gamma_{v} \vec{r} \quad \vec{r}_{c}=\vec{R}-\gamma_{c} \vec{r}, \\
\gamma_{v} & =\frac{m_{c}}{m_{c}+m_{v}} \quad \gamma_{c}=-\frac{m_{v}}{m_{c}+m_{v}} .
\end{aligned}
$$

The projectile wave functions are the eigenfunctions of $h_{p}$, and a process of discretization of the continuum is employed to treat breakup states $[2,14,16,22]$. The index $i$ is used to denote the states resulting from this discretization procedure. The $j-j$ coupling system is chosen to express the projectile wave functions,

$$
\Phi_{J_{p}}^{i}(\vec{r})=\sum_{l, j, I} \frac{\varphi_{J_{p} l j I}^{i}(r)}{r}\left[\mathcal{Y}_{l s j} \otimes \chi_{I}\right]_{J_{p}},
$$

where $\varphi_{J_{p} l j I}^{i}$ is the radial internal wave function of the projectile for a definite $l$ (orbital angular momentum of the valence-core system); $\vec{j}=\vec{l}+\vec{s}$, where $s$ is the spin of the valence particle; and $I$ is the spin of the core. $\mathcal{Y}_{l s j}$ is the spin spherical harmonic and $\chi_{I}$ is the wave function of the core (core and valence states are assumed to be completely determined by their spins).

The target wave functions $\left[\Phi_{J_{t}}^{n}(\xi)\right]$ are the eigenstates of $h_{t}$, depend on $\xi$, have an angular momentum $J_{t}$, and are completely defined by the quantum number(s) $n$.

The scattering wave functions are chosen as in Refs. [14,16,23]:

$$
\begin{aligned}
\Psi_{J_{T}, M_{T}}(\vec{R}, \vec{r}, \xi)= & \sum_{\beta} \chi_{\beta}^{J_{T}}(R) \\
& \times\left\{\left[Y_{L}(\hat{R}) \otimes \Phi_{J_{p}}^{i}(\vec{r})\right]_{J} \otimes \Phi_{J_{t}}^{n}(\xi)\right\}_{J_{T}, M_{T}},
\end{aligned}
$$

where $\beta$ denotes all the quantum numbers necessary to define the channel $\beta=\left\{L, i, J_{p}, J, J_{t}, n\right\}$. From now on we will be using a notation similar to that employed in Refs. [13,14]:

$$
\left\langle\hat{R}, \vec{r}, \xi \mid \beta, J_{T} M_{T}\right\rangle=\left\{\left[Y_{L}(\hat{R}) \otimes \Phi_{J_{p}}^{i}(\vec{r})\right]_{J} \otimes \Phi_{J_{t}}^{n}(\xi)\right\}_{J_{T}, M_{T}} .
$$

The most important physical ingredients for our calculations are the coupling potentials:

$$
U_{\beta, \beta^{\prime}}^{J_{T}}(R)=\left\langle\beta, J_{T} M_{T}\left|V_{v t}(\vec{R}, \vec{r}, \xi)+V_{c t}(\vec{R}, \vec{r}, \xi)\right| \beta^{\prime}, J_{T} M_{T}\right\rangle .
$$

It is assumed that both $V_{v t}$ and $V_{c t}$ can be expanded in multipoles of $\vec{r}$, such as

$$
V_{v t}\left(\vec{r}_{v}\right)=\sqrt{4 \pi} \sum_{Q q} V_{Q q}\left(r_{v}, \xi\right) Y_{Q q}\left(\hat{r}_{v}\right),
$$

and likewise for $V_{c t}$. In some common models for nuclear excitations, such as the rotational or vibrational models [24], it is possible to factorize the potential in radial and structure parts, leading to

$$
V_{v t}\left(\vec{r}_{v}\right)=\sqrt{4 \pi} \sum_{Q q} V_{v t}^{Q}\left(r_{v}\right) \mathcal{T}_{Q q}^{*}(\xi) Y_{Q q}\left(\hat{r}_{v}\right),
$$

where the radial part is $V_{v t}^{Q}\left(r_{v}\right)$ and $\mathcal{T}_{Q q}^{*}(\xi)$ is an operator with the same tensorial character as $Y_{Q q}$, appropriate to the structure model used to describe the target.

The coupling potentials (9) can be expressed as

$$
\begin{aligned}
U_{\beta, \beta^{\prime}}^{J_{T}}(R)= & \left\langle\beta\left\|V_{v t}+V_{c t}\right\| \beta^{\prime}\right\rangle \\
= & \sum_{\Lambda, \Lambda^{\prime}, Q}(-1)^{Q+J_{T}+J^{\prime}+J_{t}} \hat{J} \hat{J}^{\prime}\left\{\begin{array}{lll}
J^{\prime} & J_{t}^{\prime} & J_{T} \\
J_{t} & J & Q
\end{array}\right\} \\
& \times\left\{\begin{array}{lll}
L^{\prime} & J_{p}^{\prime} & J^{\prime} \\
\Lambda & \Lambda^{\prime} & Q \\
L & J_{p} & J
\end{array}\right\} \frac{1}{\sqrt{4 \pi}} \hat{L} \hat{\Lambda}\left\langle L 0 \Lambda 0 \mid L^{\prime} 0\right\rangle \\
& \times F_{\beta \beta^{\prime}}^{\Lambda, \Lambda^{\prime}, Q}(R),
\end{aligned}
$$

where $6 j, 9 j$ symbols and Clebsch-Gordan coefficients appear as usual and $\hat{A}=\sqrt{2 A+1}$. Primed quantities refer to the 
initial channel and unprimed ones to the final channel. $\Lambda, \Lambda^{\prime}$, and $Q$ are the orbital, projectile, and target angular momentum transferred, respectively, and the radial form factors $F_{\beta \beta^{\prime}}^{\Lambda, \Lambda^{\prime}, Q}(R)$ do not depend on the orbital angular momentum $L$. The expression for these form factors is the following:

$$
\begin{aligned}
F_{\beta \beta^{\prime}}^{\Lambda, \Lambda^{\prime}, Q}(R)= & (-1)^{J_{p}^{\prime}+\Lambda+J_{t}^{\prime}-J_{t}} \sqrt{4 \pi} \hat{J}_{p} \hat{J}_{p}^{\prime} \hat{l} \hat{l}^{\prime} \hat{j} \hat{j}^{\prime} \hat{\Lambda} \hat{\Lambda}^{\prime 2} \hat{Q}^{2} \sum_{\alpha, \alpha^{\prime}, K, \lambda}(-1)^{j^{\prime}+j+l+l^{\prime}+s+I} \hat{K}^{2}\left(\begin{array}{l}
Q \\
\lambda
\end{array}\right) \\
& \times\left(\begin{array}{ccc}
l^{\prime} & l & \Lambda^{\prime} \\
0 & 0 & 0
\end{array}\right)\left(\begin{array}{ccc}
\lambda & K & \Lambda \\
0 & 0 & 0
\end{array}\right)\left(\begin{array}{ccc}
Q-\lambda & K & \Lambda^{\prime} \\
0 & 0 & 0
\end{array}\right)\left\{\begin{array}{ccc}
\Lambda^{\prime} & \Lambda & Q \\
\lambda & Q-\lambda & K
\end{array}\right\}\left\{\begin{array}{ccc}
j^{\prime} & j & \Lambda^{\prime} \\
l & l^{\prime} & s
\end{array}\right\} \\
& \times\left\{\begin{array}{ccc}
J_{p}^{\prime} & J_{p} & \Lambda^{\prime} \\
j & j^{\prime} & I
\end{array}\right\} R_{\alpha, \alpha^{\prime}}^{Q, \lambda}(R) \delta_{I, I^{\prime}}\left\langle J_{t}, n\left\|\mathcal{T}_{Q}(\xi)\right\| J_{t}^{\prime}, n^{\prime}\right\rangle
\end{aligned}
$$

where $\left\langle J_{t}, n\left\|\mathcal{T}_{Q}(\xi)\right\| J_{t}^{\prime}, n^{\prime}\right\rangle$ is the reduced matrix element of $\mathcal{T}_{Q q}$ as defined in Eq. (11) between the states of the target $\left\{J_{t}, n\right\},\left\{J_{t}^{\prime}, n^{\prime}\right\} ; \alpha\left(\alpha^{\prime}\right)$ is the final (initial) state of the projectile and includes all quantum numbers necessary for the determination of the corresponding component $\alpha=\{i, l, j, I\}$,

$$
\left(\begin{array}{l}
Q \\
\lambda
\end{array}\right)=\sqrt{\frac{(2 Q) !}{[2(Q-\lambda)] !(2 \lambda) !}},
$$

and $R_{\alpha, \alpha^{\prime}}^{Q, \lambda, K}(R)$ are defined as follows:

$$
R_{\alpha, \alpha^{\prime}}^{Q, \lambda, K}(R)=\int \varphi_{J_{p}, \alpha}^{*}(r) \varphi_{J_{p}^{\prime}, \alpha^{\prime}}(r) V_{v t}^{Q K}(R, r) R^{\lambda}\left(\gamma_{v} r\right)^{Q-\lambda} d r
$$

where $\varphi_{J_{p}, \alpha}$ are as defined in Eq. (6) and $\alpha$ denotes all the necessary quantum numbers that define the projectile components. $V_{v t}^{Q K}(R, r)$ results from the multipole expansion of $V_{v t}^{Q}$, according to

$$
V_{v t}^{Q K}(R, r)=\frac{1}{2} \int_{-1}^{1} \frac{V_{v t}^{Q}\left(r_{v}\right)}{r_{v}^{Q}} P_{K}(u) d u, \quad u=\hat{R} \cdot \hat{r} .
$$

Equations similar to (15) and (16) apply to the core-target interaction, replacing $V_{v t}$ by $V_{c t}$ and $\gamma_{v}$ by $\gamma_{c}$. For the following calculations we have chosen the particle-rotor model [24] to describe the collective excitation of the target nucleus. In this model we deform a central potential $V^{(0)}(r)$ through the following transformation:

$$
V(\vec{r}, \hat{\xi})=V^{(0)}\left[r-\delta_{2} Y_{20}(\hat{\xi})\right],
$$

and we then perform an expansion in multipoles:

$$
V(\vec{r}, \hat{\xi})=\sqrt{4 \pi} \sum_{Q q} V^{Q}(r) \mathcal{D}_{q 0}^{Q}\left(\alpha^{\prime}, \beta^{\prime}, \gamma^{\prime}\right) Y_{Q q}(\hat{r})
$$

where

$$
V^{Q}(r)=\frac{\hat{Q}}{2} \int_{-1}^{1} V^{(0)}\left[r-\delta_{2} Y_{20}(\hat{\xi})\right] P_{Q}(u) d u, \quad u=\hat{r} \cdot \hat{\xi} .
$$

$\hat{\xi}$ can be interpreted as the orientation of the axis of the rotor, and $\alpha^{\prime}, \beta^{\prime}, \gamma^{\prime}$ are the Euler angles that change from the intrinsic frame of the rotor to the laboratory system and $\mathcal{D}$ is the Wigner rotation matrix.
In this model the reduced matrix element has a simple form:

$$
\begin{aligned}
\left\langle J_{t}, n\left\|\mathcal{T}_{Q}(\hat{\xi})\right\| J_{t}^{\prime}, n^{\prime}\right\rangle & =\left\langle J_{t}, K\left\|\mathcal{D}^{Q *}\right\| J_{t}^{\prime}, K\right\rangle \\
& =\hat{J}_{t}^{\prime}\left\langle J_{t}^{\prime} K Q 0 \mid J_{t} K\right\rangle
\end{aligned}
$$

It should be noted that, in general, reduced matrix elements for Coulomb and nuclear parts may differ, so it is necessary to compute the contribution of both potentials separately. However, in the particle-rotor model the reduced matrix elements are the same, while the Coulomb potential multipoles are expressed based on the reduced multipole electric operators:

$$
V^{Q}(r)=\frac{\sqrt{4 \pi}}{\hat{Q}^{2}} \frac{Z_{t} e}{r^{Q+1}} \mathcal{M}(E Q) .
$$

\section{CALCULATIONS}

In this section we present some calculations, focusing on the angular differential cross section for target excitation and its interplay with projectile breakup.

\section{A. ${ }^{58} \mathrm{Ni}(d, d)^{58} \mathrm{Ni}^{*}$}

As a test of the formalism, we have tried to reproduce the results in Ref. [20] for the reaction ${ }^{58} \mathrm{Ni}(d, d){ }^{58} \mathrm{Ni}^{*}$ at a deuteron energy of $E_{d}=80 \mathrm{MeV}$. We have followed the same procedure outlined in Ref. [20], using the same potentials and discretization procedure. The target is considered only in its ground $\left(0^{+}\right)$and first-excited $\left(2^{+}\right)$states.

The results are shown in Fig. 1 for the elastic ${ }^{58} \mathrm{Ni}(d, d){ }^{58} \mathrm{Ni}$ (g.s.) (top) and inelastic scattering ${ }^{58} \mathrm{Ni}\left(d, d^{\prime}\right)$ ${ }^{58} \mathrm{Ni}\left(2^{+}\right)$(bottom), along with those from Ref. [20] (red squares). For comparison, we include also the experimental data from Ref. [25] (circles). Since the calculations of Ref. [20] included only the $l=0$ continuum of the deuteron, we include also our calculation restricted to $l=0$ continuum (black dashed line). It is seen that the agreement between both calculations is very good, taking this as a validation of the formalism and its implementation.

In Ref. [20], the lack of $l=2$ breakup was alluded to as a possible reason for the disagreement between the elastic data and their calculation. We have checked this assumption by including $l=2$ breakup, keeping the same $V_{p n}$ potential as for the $l=0$ wave. The result (solid lines in Fig. 1) shows that 


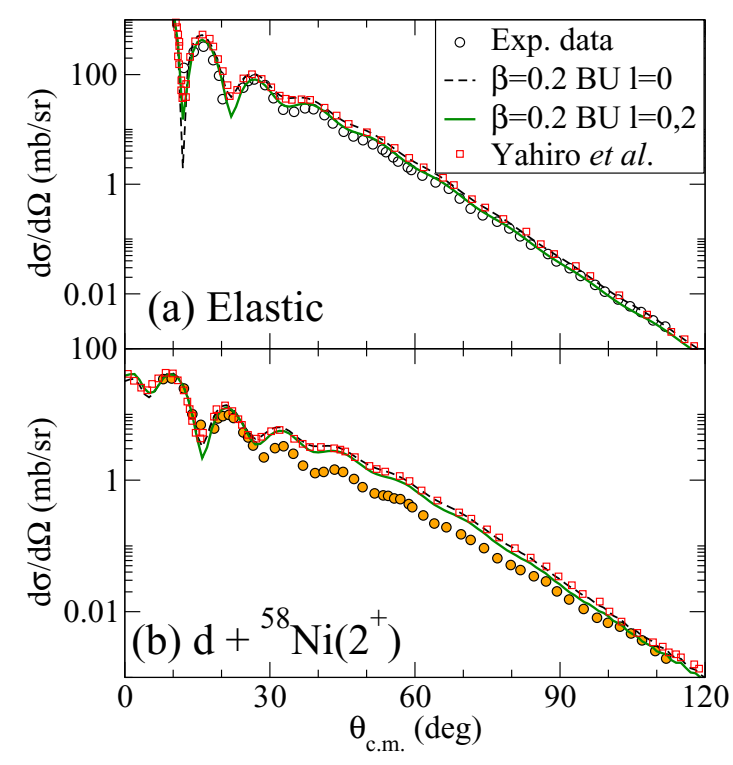

FIG. 1. (a) Elastic and (b) ${ }^{58} \mathrm{Ni}\left(2^{+}\right)$excitation angular differential cross sections for the ${ }^{58} \mathrm{Ni}(d, d)^{58} \mathrm{Ni}$ reaction at a deuteron energy of $E_{d}=80 \mathrm{MeV}$. In dashed black a calculation including only $l=0$ breakup, while in solid green the calculation includes both $l=0$ and $l=2$ breakup. Experimental data are taken from Ref. [25]. As can be seen, the agreement with the data is improved in the elastic case when including $l=2$ while for the ${ }^{58} \mathrm{Ni}\left(2^{+}\right)$excitation there is only a slight improvement in the agreement. The red squares correspond to the calculation from Ref. [20], which includes only $l=0$ breakup. As can be seen in the figure, the agreement with our $l=0$ calculation is excellent.

the agreement is indeed improved for the elastic scattering, while the inelastic cross section is only slightly modified.

We have also studied the relative importance of breakup and target excitation on the elastic cross sections, as well as the influence of breakup on the inelastic cross sections. This is illustrated in Fig. 2. In the top panel, we show the elasticscattering differential cross section calculated with the full CDCC calculation, i.e., including deuteron breakup and target excitation (solid line), and the calculations omitting either target deformation (dot-dashed) or deuteron breakup (dashed line) and that omitting both breakup and target excitation (dotted line). In the bottom panel, we compare the full CDCC calculation with the calculation omitting the breakup channels. We find that, in accord with Ref. [20], inclusion of breakup and target excitation results in a significant improvement of the agreement with the elastic-scattering data, although for the inelastic scattering cross section ${ }^{58} \mathrm{Ni}\left(2^{+}\right)$, the agreement is only slightly improved. Since this calculation has been performed as a test of the formalism, we have not pursued further accordance with experimental data.

\section{B. ${ }^{24} \mathrm{Mg}(d, d)^{24} \mathrm{Mg}^{*}$}

In this section we study the reaction ${ }^{24} \mathrm{Mg}(d, d){ }^{24} \mathrm{Mg}^{*}$ leading to the ground and first-excited states of ${ }^{24} \mathrm{Mg}$. This reaction was measured at different energies in the range of tens of $\mathrm{MeV}$ [18], from which we focus on the reaction at a deuteron

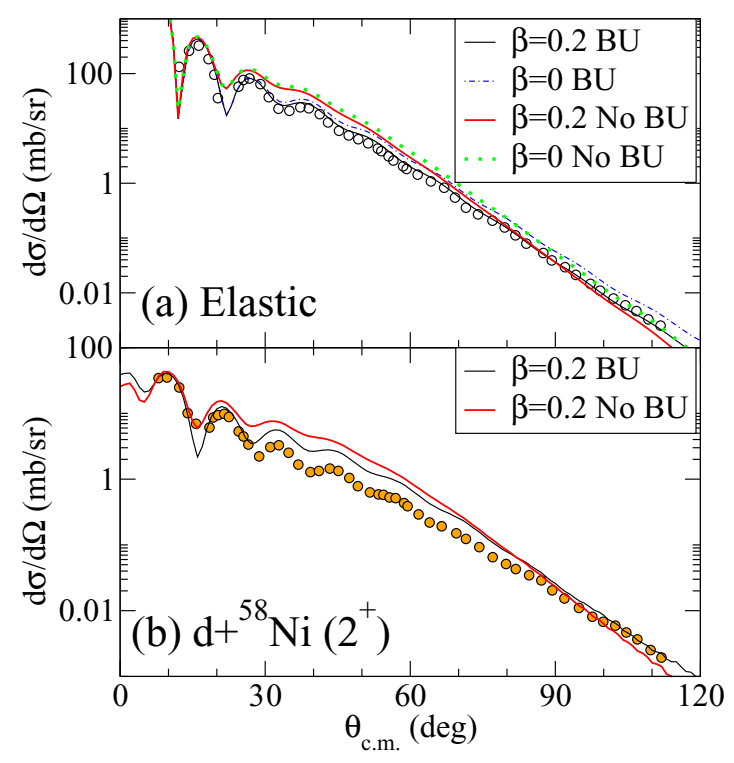

FIG. 2. Same as Fig. 1. The black solid line includes breakup and target excitation consistently. The red solid line only includes target excitation, while the blue dash-dotted line includes only breakup. The green dotted line excludes both target excitation and breakup. It can be seen that the best agreement with the data is obtained when including both breakup and inelastic excitation consistently.

energy of $70 \mathrm{MeV}$. For the deformation parameter of ${ }^{24} \mathrm{Mg}$ we have chosen a value $\beta=0.5$, in accordance with Ref. [26]. We have performed calculations using Köning-Delaroche [27] and CH89 [28] parametrizations for $p, n-{ }^{24} \mathrm{Mg}$ and the potential from Ref. [20] for the $p-n$ interaction. The results of our calculations, as well as those obtained from Faddeev calculations [26] employing the $\mathrm{CH} 89$ parametrization and the $p-n$ CD Bonn potential [29] are presented in Fig. 3. Although the calculations differ slightly, all seem to agree equally well with experimental data. All calculations underestimate the inelastic cross section at the minimum at 20 degrees, which may point to some kind of inadequacy in the nucleon-nucleus interactions used. However, the good agreement between Faddeev-AGS calculations and those presented here is taken as proof of the robustness of the CDCC results. It seems as though a smaller deformation parameter would give a better fit to the experimental data, as indicated in Ref. [26]. However, in this same paper different deformation parameters were found to give best fits to data at different deuteron energies. Therefore, in this paper we will not try to extract a value for the deformation parameter of ${ }^{24} \mathrm{Mg}$ beyond confirming the conclusions in Ref. [26].

In Ref. [26] the inclusion of higher excited states of ${ }^{24} \mathrm{Mg}$ was suggested but was not calculated due to the computational cost of the Faddeev calculation. Since the method used here is less demanding computationally than that used in Ref. [26], we have performed calculations including the $2^{+}$and $4^{+}$states of ${ }^{24} \mathrm{Mg}$, coupled through quadrupolar and hexadecapolar deformation $\beta_{4}=-0.017$ [30]. The results are shown in Fig. 4. The effect of the $4^{+}$state is found to be rather small, being unnoticeable in the elastic cross section and leading to a small 


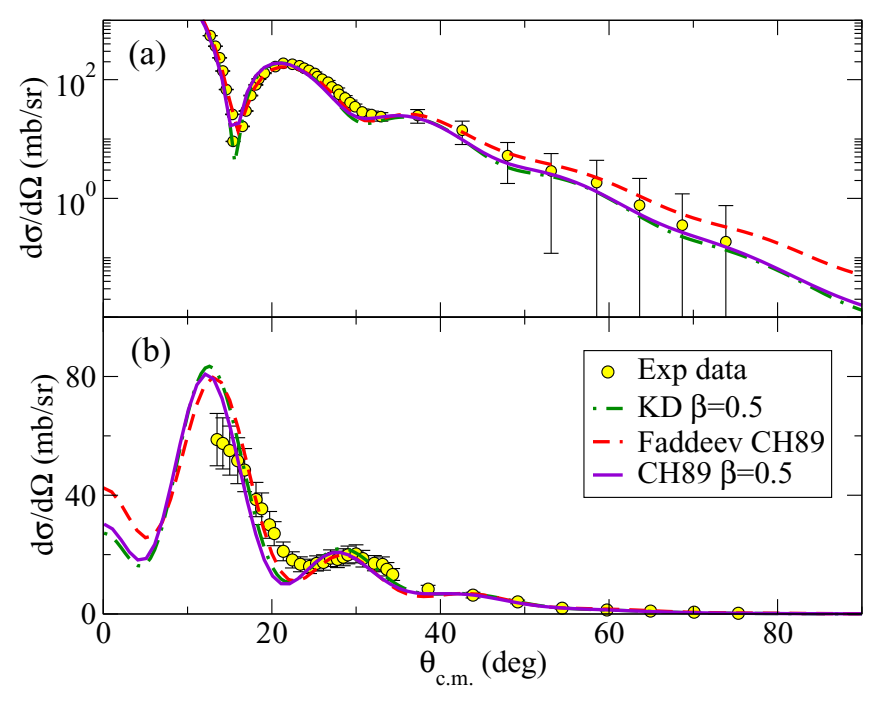

FIG. 3. Elastic (upper) and ${ }^{24} \mathrm{Mg}\left(2^{+}\right)$excitation (lower) differential angular cross sections for ${ }^{24} \mathrm{Mg}\left(d, d^{\prime}\right)^{24} \mathrm{Mg}^{*}$ at a deuteron energy of $70 \mathrm{MeV}$. CDCC calculations are presented using KöningDelaroche and $\mathrm{CH} 89$ parametrizations. Faddeev calculations are taken from Ref. [26].

reduction of the cross section at the peak of the inelastic cross section.

\section{C. ${ }^{144} \mathrm{Sm}\left({ }^{6} \mathrm{Li},{ }^{6} \mathrm{Li}\right){ }^{144} \mathrm{Sm}^{*}$}

In this section we study the inelastic scattering of ${ }^{6} \mathrm{Li}$ from ${ }^{144} \mathrm{Sm}$ which has been recently measured at energies around the Coulomb barrier [19]. This reaction has been previously studied [19] by decoupling target excitation and breakup of the projectile. First, CDCC was used to calculate breakup and a folding potential was obtained from this calculation. This

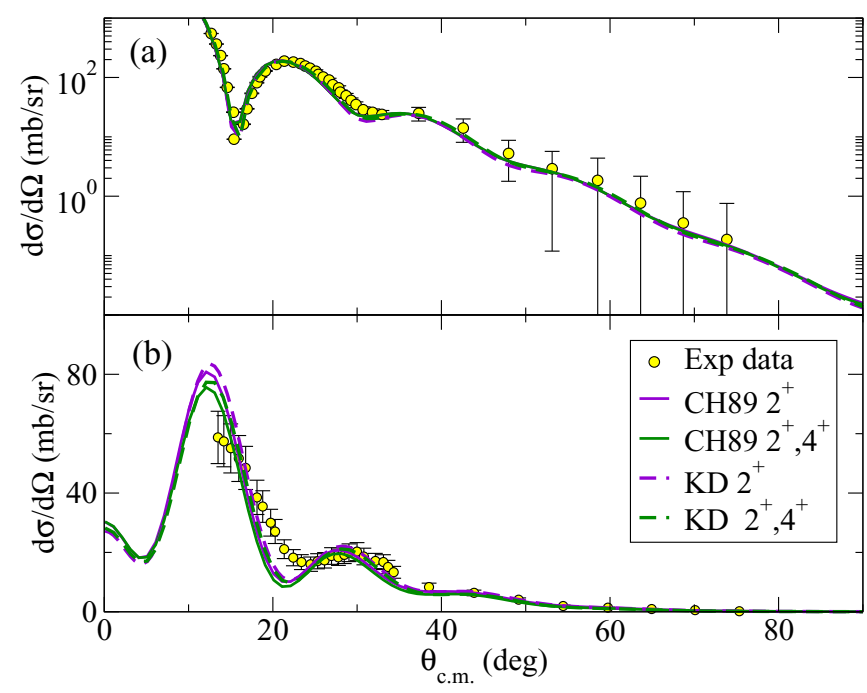

FIG. 4. Elastic (upper) and ${ }^{24} \mathrm{Mg}\left(2^{+}\right)$excitation (lower) differential angular cross sections for ${ }^{24} \mathrm{Mg}\left(d, d^{\prime}\right){ }^{24} \mathrm{Mg}^{*}$ at a deuteron energy of $70 \mathrm{MeV}$ computed including (violet lines) and excluding (green lines) the $4^{+}$state of ${ }^{24} \mathrm{Mg}$, using CH89 (solid lines) and Köning-Delaroche (dashed lines) parametrizations. potential was then deformed to account for target excitation. Due to this decoupling, target excitation and breakup of the projectile are not treated consistently. Since ${ }^{6} \mathrm{Li}$ is relatively weakly bound, its breakup is an important process that can couple to the excitation of the collective degrees of freedom of ${ }^{144} \mathrm{Sm}$, so a consistent treatment of both effects might be of importance to describe this reaction. The extension of CDCC described in Sec. II allows us to treat both processes on equal footing. Therefore, we find its application to this reaction to be suitable and relevant.

We focus on the elastic and inelastic $\left[{ }^{144} \mathrm{Sm}\left({ }^{6} \mathrm{Li}\right.\right.$, $\left.\left.{ }^{6} \mathrm{Li}\right){ }^{144} \mathrm{Sm}^{*}\right]$ angular differential cross section as our observables of interest. Experimental data exist for four different incoming ${ }^{6} \mathrm{Li}$ energies: $23,28,30$, and $35 \mathrm{MeV}$. This allows for a certain systematic study of the results obtained.

For the following calculations we have considered ${ }^{6} \mathrm{Li}$ to be a binary system composed of an inert deuteron and an inert $\alpha$ particle, so that their excitations and possible breakup are neglected. For ${ }^{144} \mathrm{Sm}$ only the first $2^{+}$and $3^{-}$states have been considered, with excitation energies of 1.66 and $1.81 \mathrm{MeV}$, respectively. The deformation parameters $\beta_{2}=0.087$ and $\beta_{3}=0.130$ [19] have been used to deform the $d / \alpha-{ }^{144} \mathrm{Sm}$ potentials. The reduced Coulomb matrix elements have been computed from the $B(E \lambda)$ probabilities [see, e.g., Eq. (4.4.3) from Ref. [31] ]:

$$
B\left(E \lambda, I_{i} \rightarrow I_{f}\right)=\frac{1}{2 I_{i}+1}\left|\left\langle I_{f}\|\mathcal{M}(E \lambda)\| I_{i}\right\rangle\right|^{2},
$$

using the $B\left(E \lambda, I_{i} \rightarrow I_{f}\right)$ values from Refs. [32,33].

The continuum of ${ }^{6} \mathrm{Li}$ has been discretized by using a transformed harmonic-oscillator basis [34] extending up to $8 \mathrm{MeV}$ and considering only the $s$ and $d$ waves. For the $\alpha-d$ interaction we have used the potential of Ref. [35], which includes the deuteron spin and gives a proper description of both the ground state and the $3^{+}$resonance of ${ }^{6} \mathrm{Li}$. Due to the experimental impossibility of distinguishing between the $2^{+}$ and $3^{-}$states of ${ }^{144} \mathrm{Sm}$, the inelastic cross sections presented are the sum of the contributions for both states.

For the $d-{ }^{144} \mathrm{Sm}$ and $\alpha-{ }^{144} \mathrm{Sm}$ potentials different parametrizations have been used. In Fig. 5 we present calculations employing two different sets of potentials.

The red dashed line (Set 1) potential has been constructed using the São Paulo parametrization [36], both for the $\alpha-{ }^{144} \mathrm{Sm}$ and $d-{ }^{144} \mathrm{Sm}$ interactions. Since the energies considered are not too different, the dependence of the potential on the incident energy has been neglected. The imaginary part of the potential was rescaled by a factor $N_{i \alpha}$ for the $\alpha-{ }^{144} \mathrm{Sm}$ potential and by a factor $N_{i d}$ for the $d-{ }^{144} \mathrm{Sm}$ potential, in order to reproduce the elastic-scattering data. A fairly negligible dependence on $N_{i \alpha}$ was found, therefore a value of $N_{i \alpha}=1$ was chosen. For the $d-{ }^{144} \mathrm{Sm}$ potential, a factor of $N_{i d}=0.2$ was found to give a reasonable agreement for all energies considered.

The solid blue line (Set 2) corresponds to a calculation in which the $\alpha{ }^{144} \mathrm{Sm}$ potential is based once again on the São Paulo parametrization. The imaginary potential has been rescaled by using a factor of $N_{i \alpha}=0.78$ this time. Meanwhile, for the $d-{ }^{144} \mathrm{Sm}$ potential, we have chosen Perey-Perey global potential [37], whose imaginary part has been rescaled by using a factor of $N_{i d}=0.7$. This reduction of the imaginary potential 


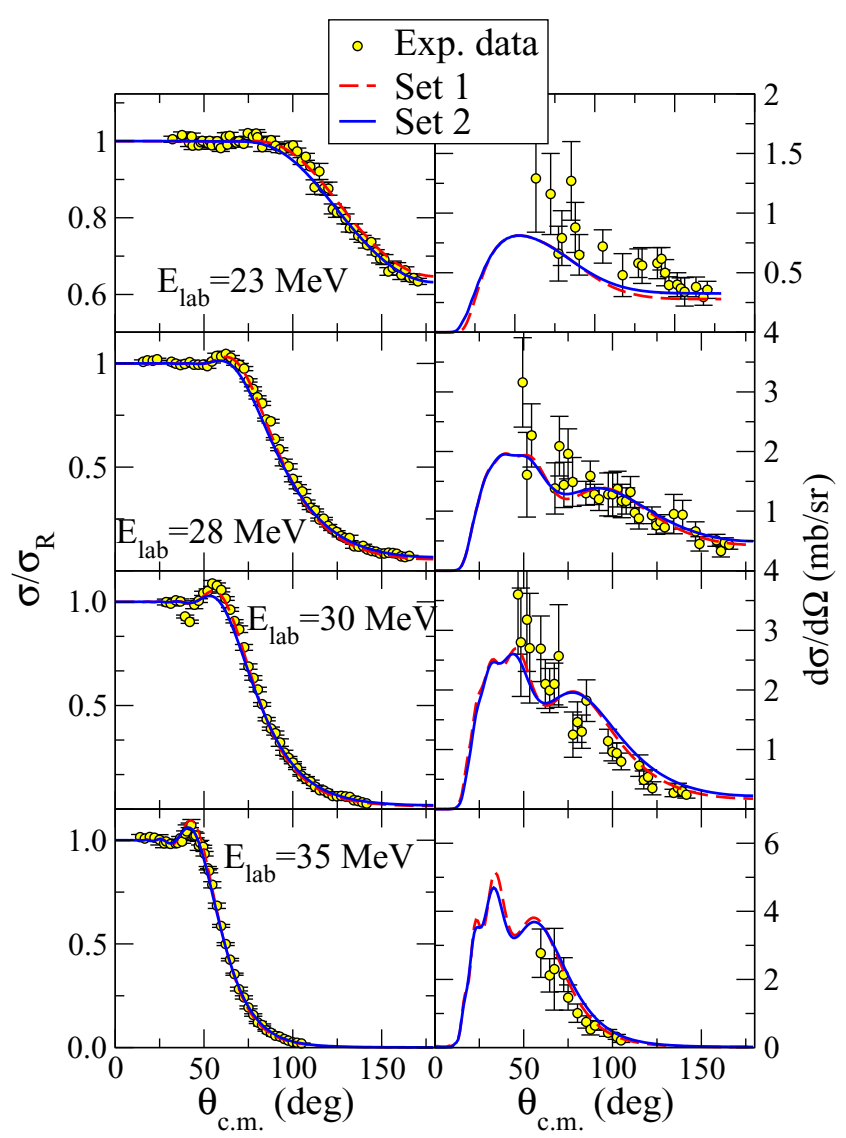

FIG. 5. Elastic (left) and ${ }^{144} \mathrm{Sm}$ excitation (right) differential angular cross sections for ${ }^{144} \mathrm{Sm}\left({ }^{6} \mathrm{Li},{ }^{6} \mathrm{Li}\right){ }^{144} \mathrm{Sm}^{*}$ for incoming energies of $23,28,30$, and $35 \mathrm{MeV}$. The red dashed and solid blue lines correspond to different $\alpha-{ }^{144} \mathrm{Sm}$ and $d-{ }^{144} \mathrm{Sm}$ potentials (see text).

for the deuteron inside ${ }^{6} \mathrm{Li}$ has been discussed previously [38-41]. We find this selection of potentials to give a good fit to the data at all considered incident energies.

In general we find the inelastic cross section to be quite insensitive to the selection of potentials, while Set 1 seems to give a better agreement with the elastic data around the Coulomb-nuclear interference peak.

We find that the inclusion of target excitation in the calculations gives a small contribution to the elastic cross section. This can be seen in Fig. 6, where the solid red line corresponds to calculations using potentials from Set 1 , described above, including deformation of the target. Meanwhile, the blue dashed line represents calculations using the same potentials setting the deformation parameter to 0 . As can be seen, both calculations give very similar results.

To study the effect of breakup in both elastic and inelastic cross sections we have performed a calculation excluding all breakup states from the coupled-channel calculation, for which we have chosen the potentials from Set 2. The results are shown in Fig. 7, the solid blue line corresponding to the CDCC calculation including breakup and the green dash-dotted line to that excluding it. As can be seen in the figure, without breakup, the elastic differential cross section is underestimated at all the energies at larger angles, probably due to the exclusion

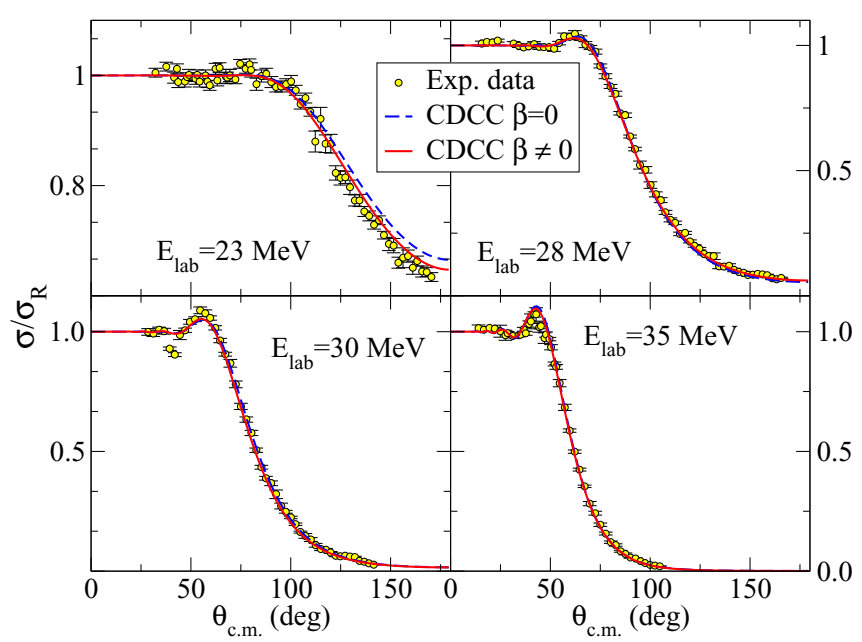

FIG. 6. Elastic angular differential cross section for different incident energies for the reaction ${ }^{144} \mathrm{Sm}\left({ }^{6} \mathrm{Li},{ }^{6} \mathrm{Li}\right){ }^{144} \mathrm{Sm}$. The red solid line corresponds to calculations using potentials from Set 1 (see text) including deformation of the target, while the blue dashed line corresponds to calculations using the same potentials without target deformation.

of the resonance at $E_{d \alpha}=0.7 \mathrm{MeV}$. As for the inelastic cross section, it is found that the effect of breakup is only moderate, smoothing the oscillation of the cross section, but not modifying its magnitude much. It can be seen that the effect of the breakup states becomes more important with increasing incident energy but even for the highest energy the agreement with the data of both calculations is similar.

Since in this particular case the effect of projectile breakup in target excitation is small, it is plausible to anticipate that this effect can be accounted for by using an optical model description of the elastic channel. To assess this, we have performed a coupled-channel calculation in which the incident channel is described by means of an optical potential and so breakup effects are only accounted for effectively. We have used the Cook potential [42], which we have deformed employing the deformation parameters indicated above, assuming a rotor model for ${ }^{144} \mathrm{Sm}$. The results are shown in Fig. 7 in order to compare it with the full CDCC calculation and the calculation without breakup. It is rather remarkable the good agreement that is obtained for the inelastic cross section between the full CDCC calculation and the optical model one: the curves overlap except for a small increase at intermediate angles for the optical model result. Let us remark that CDCC and optical model calculations use potentials from completely different systematics, the only point in common being the deformation parameters and the rotational model used for the deformation. In the elastic cross section we can see that the optical model calculation seems to underestimate the data at the large-angle tail, probably due to an inadequate treatment of the ${ }^{6} \mathrm{Li} 3^{+}$resonance. This result conclusively indicates that, for this reaction, the main effect of breakup states on the ${ }^{144} \mathrm{Sm}$ excitation cross section is to remove flux from the incoming channel in a way that can be adequately described with an optical potential. 


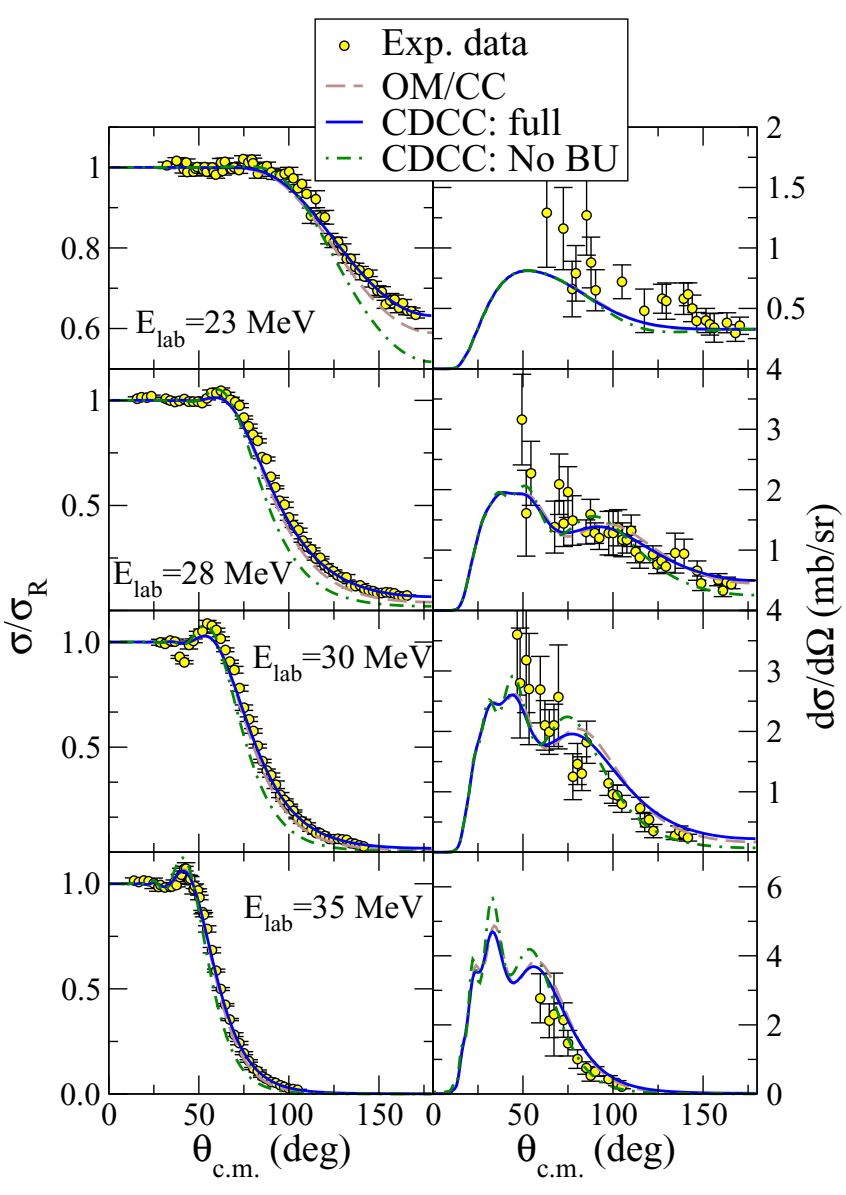

FIG. 7. Elastic (left) and ${ }^{144} \mathrm{Sm}$ excitation (right) differential cross sections for ${ }^{144} \mathrm{Sm}\left({ }^{6} \mathrm{Li},{ }^{6} \mathrm{Li}\right){ }^{144} \mathrm{Sm}^{*}$ at different incident energies. Coupled-channel calculations are presented using potentials from Set 2 (see text) including (solid blue line) and excluding (dash-dotted green line) breakup states from the calculation. The dashed brown line corresponds to optical model calculations using the Cook potential [42].

With the aim of obtaining a deeper understanding of the similitude of the calculated inelastic cross sections for both potential sets for the CDCC calculation and for the optical model potential we have plotted in Fig. 8 the radial form factors $F_{\text {elas, inel }}^{2,0,2}(R)$ corresponding to the ${ }^{6} \mathrm{Li}$ (g.s. $)+$ ${ }^{144} \mathrm{Sm}\left(0^{+}\right) \rightarrow{ }^{6} \mathrm{Li}$ (g.s. $)+{ }^{144} \mathrm{Sm}\left(2^{+}\right)$target excitation for the CDCC calculations as well as for the deformed Cook potential. The shaded region corresponds to the range of distances of closest approach, assuming a classical Coulomb trajectory, for scattering angles between 50 and 150 degrees (which corresponds to the angular range spanned by the data) at an incident energy of $30 \mathrm{MeV}$. It is seen that, within this radial range, the coupling potential is dominated by the its real part, which turns out to be very similar in the three cases. Much larger differences are observed in the nuclear interior (see inset) but, due to the combined Coulomb and centrifugal barriers, the direct reaction channels will not be sensitive to these short distances. This may explain why different potential sets (even the considered deformed optical model potential) give rise to similar inelastic cross sections. From the calculated

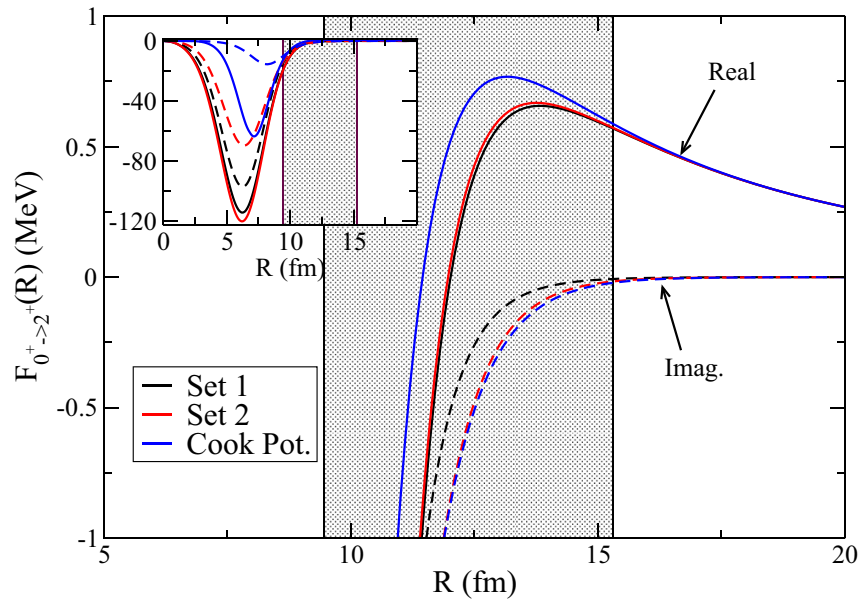

FIG. 8. Coupling potential $F_{\text {elas,inel }}^{2,0,2}$ for the ${ }^{6} \mathrm{Li}(\mathrm{g} . \mathrm{s})+$. ${ }^{144} \mathrm{Sm}\left(0^{+}\right) \rightarrow{ }^{6} \mathrm{Li}$ (g.s. $)+{ }^{144} \mathrm{Sm}\left(2^{+}\right)$inelastic transition, computed from CDCC calculation with Sets 1 and 2 and with the deformed ${ }^{6} \mathrm{Li}+{ }^{144} \mathrm{Sm}$ potential, using Cook's parametrization. An incident energy of $30 \mathrm{MeV}$ has been considered. The shaded area corresponds to the distances of closest approach corresponding to Coulomb trajectories leading to scattering angles from $50^{\circ}$ to $150^{\circ}$, the range spanned by the considered experimental data [19].

differential cross sections (Fig. 7), one can see that the largest differences appear at backward angles, which correspond to the smallest projectile-target separations and hence to an enhanced sensitivity to the nuclear interior.

\section{D. ${ }^{9} \mathrm{Be}\left({ }^{6} \mathrm{Li},{ }^{6} \mathrm{Li}\right){ }^{9} \mathrm{Be}^{*}$}

In this last section, we have performed calculations for the reaction ${ }^{9} \mathrm{Be}\left({ }^{6} \mathrm{Li},{ }^{6} \mathrm{Li}\right){ }^{9} \mathrm{Be}$ at an incident energy of $20 \mathrm{MeV}$. We present calculations for the cross sections of elastic scattering; excitation to the $5 / 2^{-}$resonant state of ${ }^{9} \mathrm{Be}$ at $E_{x}=2.43 \mathrm{MeV}$, and excitation to the resonant state of ${ }^{6} \mathrm{Li}$ with $E_{x}=2.19 \mathrm{MeV}$ and $J^{\pi}=3^{+}$, for which experimental data exist [43].

Notice that, in this case, excitation to the $5 / 2^{-}$state of ${ }^{9} \mathrm{Be}$ does actually correspond to breakup, but in order to accommodate this reaction into our three-body reaction model, we treat this state as a collective excitation, assuming a rotor model for ${ }^{9} \mathrm{Be}$ and a deformation length of $\delta_{2}=2.5 \mathrm{fm}$, following Ref. [43]. ${ }^{6} \mathrm{Li}$ is treated as a binary system composed of a deuteron and an $\alpha$ particle as in the previous section.

The $\alpha-d$ interaction is the same as in the previous section. As for the $\alpha-{ }^{9} \mathrm{Be}$ interaction, the potential from Ref. [44] has been chosen. Due to the difficulty of including the spin-orbit of the fragment-target interaction in the CDCC calculations, the $\alpha-{ }_{-}^{9} \mathrm{Be}$ spin-orbit term has been applied to the whole ${ }^{6} \mathrm{Li}-{ }^{9} \mathrm{Be}$ system, following the prescription of Ref. [45].

Finally, the $d-{ }^{9} \mathrm{Be}$ potential has been obtained by folding the ground state of the deuteron with $p-{ }^{9} \mathrm{Be}$ and $n-{ }^{9} \mathrm{Be}$ interactions. For the $p-{ }^{9} \mathrm{Be}$ interaction the potential of Ref. [46] has been used. Although this potential was derived for neutrons, we found a small dependence of the observables on it. Therefore, this prescription has been chosen for convenience. For the $n-{ }^{9} \mathrm{Be}$ potential, two prescriptions have been used. In Fig. 9, the magenta dashed line corresponds to $V_{n^{9}}$ Be from Ref. [46], 


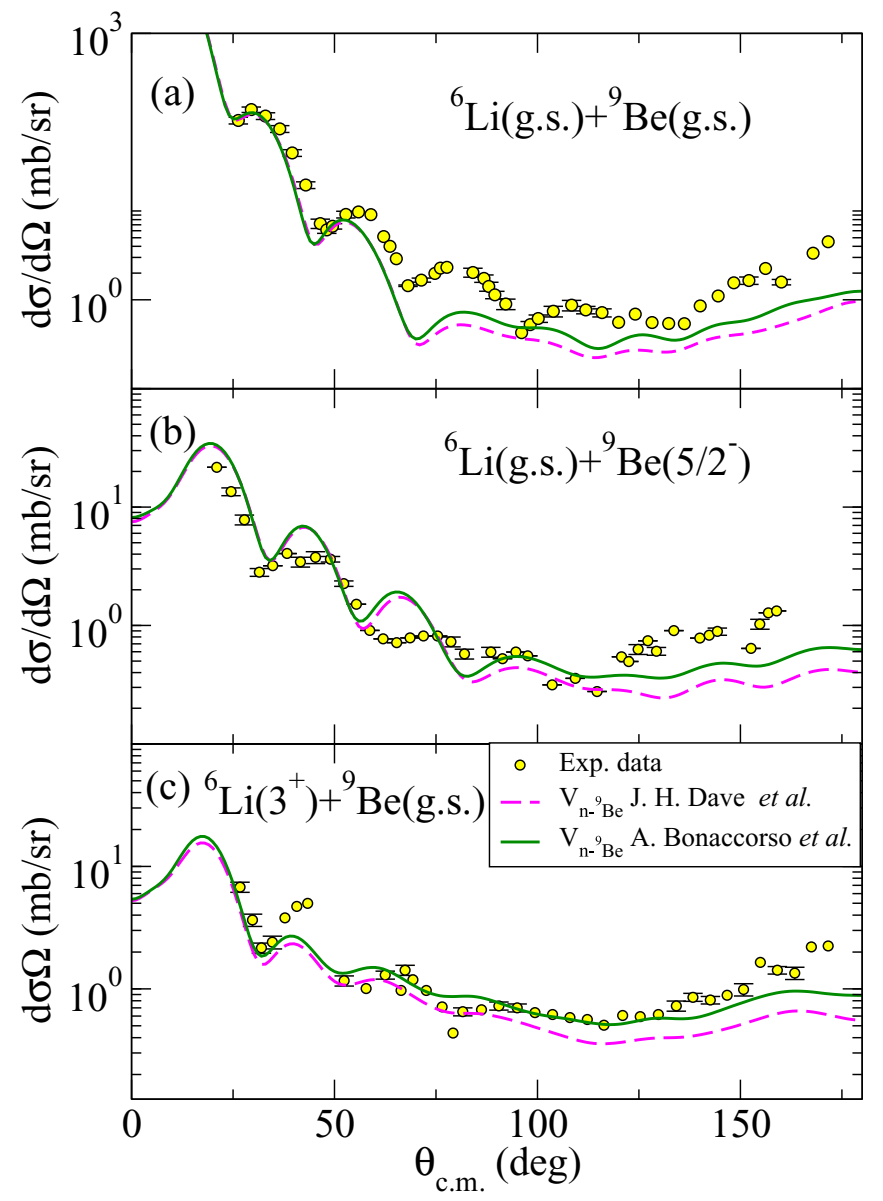

FIG. 9. Cross sections for (a) elastic scattering and (b) excitation of ${ }^{9} \mathrm{Be}\left(E_{x}=2.43 \mathrm{MeV}, J^{\pi}=5 / 2^{-}\right)$and of (c) ${ }^{6} \mathrm{Li}$ (resonant state at $E_{x}=2.19 \mathrm{MeV}, J^{\pi}=3^{+}$). Two sets of potentials are presented (see text).

while the green solid line corresponds to the parametrization from Ref. [47], which gives a more precise description of the $n-{ }^{9} \mathrm{Be}$ system at low energies. It is seen that both potentials give similar cross sections up to $50^{\circ}-60^{\circ}$, but differ afterwards finding that the potential from Ref. [47] gives a better agreement with the experimental data.

We find a reasonable agreement between the calculations and the experimental data for the excitation of ${ }^{6} \mathrm{Li}$ while the calculation for excitation of ${ }^{9} \mathrm{Be}$ presents a structure which is not seen in the data, although the magnitude of the cross section is properly reproduced. It is also found that our calculations underestimate the elastic-scattering data for larger angles.

It must be noted that this is a very challenging reaction since both projectile and target nuclei are weakly bound and display a three-body structure $\left(\alpha+p+n\right.$, for ${ }^{6} \mathrm{Li}$ and $\alpha+\alpha+n$ for ${ }^{9} \mathrm{Be}$ ) so a proper treatment of this reaction would require, at least, a six-body reaction model. Clearly, this is beyond the capabilities of our reaction model. Moreover, the excited state of ${ }^{9} \mathrm{Be}$ included in our calculations $\left(5 / 2^{-}\right)$ is actually unbound so its approximation by a discrete collective (rotational) state is only a simple way of modeling

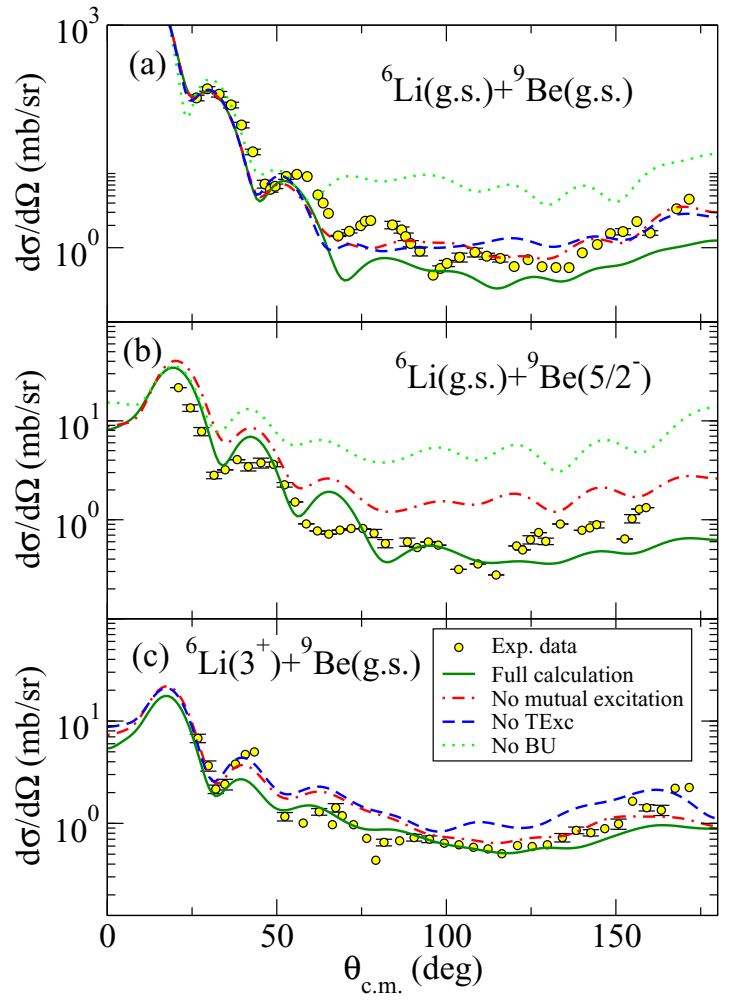

FIG. 10. Cross sections for (a) elastic scattering and (b) inelastic scattering of ${ }^{9} \mathrm{Be}\left(E_{x}=2.43 \mathrm{MeV}, J^{\pi}=5 / 2^{-}\right)$and of $(\mathrm{c}){ }^{6} \mathrm{Li}$ (resonant state at $E_{x}=2.19 \mathrm{MeV}, J^{\pi}=3^{+}$). Calculations have been performed using the potential from [47] for $V_{n-}{ }^{9} \mathrm{Be}$. The rest of the potentials are those indicated in the text. The dark-green solid line corresponds to the full calculation, while the red dot-dashed line excludes states in which ${ }^{9} \mathrm{Be}$ is in its excited state simultaneously with ${ }^{6} \mathrm{Li}$ being in a breakup state. The blue dashed line excludes all states with ${ }^{9} \mathrm{Be}$ in its excited state while the green dotted line excludes all breakup states of ${ }^{6} \mathrm{Li}$.

this complicated reaction in terms of a three-body reaction problem. Furthermore, as shown in Ref. [43], compound nucleus and heavy-cluster transfer might play a significant role in the studied observables. All these factors make deviations from the data not unexpected. Despite these limitations, we consider this to be a meaningful example, due to the existence of experimental data for the excitation of both projectile $\left({ }^{6} \mathrm{Li}\right)$ and target $\left({ }^{9} \mathrm{Be}\right)$ nuclei, so these two excitation mechanisms can be studied simultaneously in the present framework, of course, within our assumed three-body reaction model.

To study the effect of breakup, target excitation and their interplay we have performed calculations excluding different channels. The results are shown in Fig. 10. In it the dark-green solid line corresponds to the full calculation, while for the red dot-dashed line the states in which ${ }^{9} \mathrm{Be}$ is in its excited state and ${ }^{6} \mathrm{Li}$ is in a continuum state at the same time have been excluded. For the dashed blue line, the excitation of ${ }^{9} \mathrm{Be}$ has been omitted while for the dotted green line no breakup states of ${ }^{6} \mathrm{Li}$ have been included. 
It is remarkable that the effect of the states where both ${ }^{9} \mathrm{Be}$ and ${ }^{6} \mathrm{Li}$ are excited (which we may call mutual excitation) is quite important for all cross sections presented, as can be seen on the red dot-dashed line, which gives an increase in all cross sections, particularly in the cross section corresponding to the excitation of ${ }^{9} \mathrm{Be}$. While the effect of the mutual-excitation states would be expected to be small, it is however found to be quite relevant to the cross section, smaller than the effect of the direct breakup of ${ }^{6} \mathrm{Li}$ (as can be seen comparing the green dotted and red dot-dashed lines) but quite more relevant than the excitation of ${ }^{9} \mathrm{Be}$ without simultaneous breakup of ${ }^{6} \mathrm{Li}$, as is highlighted by the similarity between the red dot-dashed and blue dashed lines.

\section{SUMMARY AND CONCLUSIONS}

The extension of the CDCC method to include excitation of collective degrees of freedom of the target has been revisited without any approximation apart from those inherent to the CDCC method. The formalism has been implemented in a computer code and applied to a number of reactions, induced by deuterons and ${ }^{6} \mathrm{Li}$.

For the reaction $d+{ }^{24} \mathrm{Mg}$ our results reproduce very well those obtained with the more sophisticated Faddeev-AGS method. For ${ }^{6} \mathrm{Li}+{ }^{144} \mathrm{Sm}$ the calculations reproduce very nicely the inelastic cross sections at four different energies, although the reproduction of the elastic-scattering data required a readjustment of the deuteron-target potential, in accordance to previous findings. Furthermore, the ${ }^{6} \mathrm{Li}$ breakup was found to play only a minor role on the inelastic cross sections and these effects seem to be equally well accounted for using a optical-model description of the elastic channel. Finally, for the reaction ${ }^{6} \mathrm{Li}+{ }^{9} \mathrm{Be}$ the effects of the interplay of target excitation and projectile breakup seem to be more important than in the previous reactions, probably due to the lighter masses and the lower beam energy, which leads to a longer interaction time, although the agreement with experimental data is far from perfect.

The method developed here has potential applications in several problems of timely interest. For example, it could be used to study the simultaneous influence of projectile breakup and collective excitations of the target on fusion and transfer cross sections in reactions induced by weakly bound projectiles on deformed targets.

\section{ACKNOWLEDGMENTS}

We are thankful to A. Deltuva for providing the Faddeev calculations for Fig. 3 and to J. Lubian for providing the São Paulo potentials used in Sec. IIIC. We would also like to thank A. Di Pietro for fruitful discussions. This work has been partially supported by the European Union's Horizon 2020 research and innovation program under Grant Agreement No. 654002, by Junta de Andalucía (FQM160, P07-FQM-02894) and by the Spanish Ministerio de Economía y Competitividad (FIS2014-53448-C2-1-P). M.G.-R. acknowledges a research grant by the Spanish Ministerio de Educación, Cultura y Deporte, FPU Research Grant FPU13/04109.

\section{APPENDIX: $Q=0$ REDUCTION}

In this Appendix, we calculate the expressions corresponding to $Q=0$ from the formulas presented in Sec. II and prove that they reduce to the standard CDCC expressions without target excitation as can be found for example in Ref. [23]. It must be noted that when $Q=0$ there is no target excitation but the projectile can still break in this formalism.

We start by noting that Eq. (16) and (15) are reduced to

$$
\begin{aligned}
& V_{v t}^{0 K}(R, r)=\frac{1}{2} \int_{-1}^{1} V_{v t}\left(r_{v}\right) P_{K}(u) d u, \\
& R_{\alpha, \alpha^{\prime}}^{0,0, K}(R)=\int \varphi_{J_{p}, \alpha}^{*}(r) V_{v t}^{0 K}(R, r) \varphi_{J_{p}^{\prime}, \alpha^{\prime}}(r) d r,
\end{aligned}
$$

using the notation described in Sec. II and the fact that $\lambda \leqslant Q$.

Next we perform the reduction of Eq. (13) using the properties of $3 j$ and $6 j$ symbols:

$$
\begin{aligned}
\left(\begin{array}{lll}
0 & A & B \\
0 & 0 & 0
\end{array}\right) & =(-1)^{A} \frac{\delta_{A B}}{\hat{A}}, \\
\left\{\begin{array}{lll}
A & B & C \\
D & E & 0
\end{array}\right\} & =(-1)^{A+B+C} \frac{\delta_{A E} \delta_{B D}}{\hat{A} \hat{B}},
\end{aligned}
$$

and the reduction of Eq. (20)

$$
\left\langle J_{t}, n\left\|\mathcal{T}_{0}(\hat{\xi})\right\| J_{t}^{\prime}, n^{\prime}\right\rangle=\hat{J}_{t}^{\prime}\left\langle J_{t}^{\prime} K 00 \mid J_{t} K\right\rangle=\hat{J}_{t}^{\prime} \delta_{J_{t}^{\prime} J_{t}},
$$

which also indicates that for every model used to characterize the target we must require $\left\langle J_{t}, n\left\|\mathcal{T}_{0}(\hat{\xi})\right\| J_{t}^{\prime}, n^{\prime}\right\rangle=\hat{J}_{t}^{\prime} \delta_{J_{t}^{\prime} J_{t}}$. The reduction yields

$$
\begin{aligned}
F_{\beta \beta^{\prime}}^{K}(R)= & (-1)^{J_{p}^{\prime}+K} \sqrt{4 \pi} \delta_{\Lambda, \Lambda^{\prime}, K} \hat{J}_{p} \hat{J}_{p}^{\prime} \hat{l} \hat{l}^{\prime} \hat{j} \hat{j}^{\prime} \hat{K}^{2} \hat{J}_{t}^{\prime} \\
& \times \sum_{\alpha, \alpha^{\prime}}(-1)^{j^{\prime}+j+l+l^{\prime}+s+I}\left(\begin{array}{ccc}
l^{\prime} & l & K \\
0 & 0 & 0
\end{array}\right) \\
& \times\left\{\begin{array}{ccc}
j^{\prime} & j & K \\
l & l^{\prime} & s
\end{array}\right\}\left\{\begin{array}{ccc}
J_{p}^{\prime} & J_{p} & K \\
j & j^{\prime} & I
\end{array}\right\} R_{\alpha, \alpha^{\prime}}^{0,0, K}(R) \delta_{I, I^{\prime}} .
\end{aligned}
$$

Now for the reduction of Eq.(16) we need the reduction of $9 j$ symbols:

$$
\left\{\begin{array}{ccc}
A & B & C \\
D & E & 0 \\
F & G & H
\end{array}\right\}=(-1)^{G+A+C+D} \frac{\delta_{D E} \delta_{C H}}{\hat{D} \hat{C}}\left\{\begin{array}{lll}
A & B & C \\
G & F & D
\end{array}\right\},
$$

leading to

$$
\begin{aligned}
U_{\beta, \beta^{\prime}}^{J_{T}}(R)= & \sum_{K, K, 0} \delta_{J J^{\prime}} \delta_{J_{t} J_{t}^{\prime}} \delta_{\Lambda, \Lambda^{\prime}, K}(-1)^{J_{p}+J+L^{\prime}+L} \hat{L} \hat{L}^{\prime} \\
& \times \frac{1}{\sqrt{4 \pi}}\left\{\begin{array}{lll}
L & J_{p} & J \\
J_{p}^{\prime} & L^{\prime} & K
\end{array}\right\}\left(\begin{array}{ccc}
L & L^{\prime} & K \\
0 & 0 & 0
\end{array}\right) F_{\beta \beta^{\prime}}^{K}(R) .
\end{aligned}
$$

Both of these expressions can be shown to be equivalent to those found in Ref. [23], although the comparison is not straightforward and requires some angular-momentum algebra. 
[1] G. H. Rawitscher, Phys. Rev. C 9, 2210 (1974).

[2] N. Austern, Y. Iseri, M. Kamimura, M. Kawai, G. Rawitscher, and M. Yahiro, Phys. Rep. 154, 125 (1987).

[3] P. Banerjee and R. Shyam, Phys. Rev. C 61, 047301 (2000).

[4] J. A. Tostevin, S. Rugmai, and R. C. Johnson, Phys. Rev. C 57, 3225 (1998).

[5] L. D. Faddeev, Zh. Eksp. Teor. Fiz. 39, 1459 (1960) [Sov. Phys. JETP 12, 1014 (1961)].

[6] E. O. Alt, P. Grassberger, and W. Sandhas, Nucl. Phys. B 2, 167 (1967).

[7] S. Typel and G. Baur, Phys. Rev. C 50, 2104 (1994).

[8] H. Esbensen and G. Bertsch, Nucl. Phys. A 600, 37 (1996).

[9] T. Kido, K. Yabana, and Y. Suzuki, Phys. Rev. C 50, R1276 (1994).

[10] S. Typel and G. Baur, Phys. Rev. C 64, 024601 (2001).

[11] P. Capel, G. Goldstein, and D. Baye, Phys. Rev. C 70, 064605 (2004).

[12] A. García-Camacho, A. Bonaccorso, and D. Brink, Nucl. Phys. A 776, 118 (2006).

[13] N. C. Summers, F. M. Nunes, and I. J. Thompson, Phys. Rev. C 74, 014606 (2006).

[14] R. de Diego, J. M. Arias, J. A. Lay, and A. M. Moro, Phys. Rev. C 89, 064609 (2014).

[15] A. Deltuva, Phys. Rev. C 88, 011601 (2013).

[16] A. M. Moro and J. A. Lay, Phys. Rev. Lett. 109, 232502 (2012).

[17] J. A. Lay, R. de Diego, R. Crespo, A. M. Moro, J. M. Arias, and R. C. Johnson, Phys. Rev. C 94, 021602 (2016).

[18] A. Kiss, O. Aspelund, G. Hrehuss, K. Knpfle, M. Rogge, U. Schwinn, Z. Seres, P. Turek, and C. Mayer-Bricke, Nucl. Phys. A 262, 1 (1976).

[19] A. Woodard, J. Figueira, D. Otomar, J. F. Niello, J. Lubian, A. Arazi, O. Capurro, P. Carnelli, L. Fimiani, G. Mart et al., Nucl. Phys. A 873, 17 (2012).

[20] M. Yahiro, Y. Iseri, H. Kameyama, M. Kamimura, and M. Kawai, Prog. Theor. Phys. Suppl. 89, 32 (1986).

[21] H.-T. Pierre Chau, Eur. Phys. J. A 51, 1 (2015).

[22] J. A. Lay, A. M. Moro, J. M. Arias, and J. Gómez-Camacho, Phys. Rev. C 85, 054618 (2012).

[23] I. J. Thompson, Comput. Phys. Rep. 7, 167 (1988).

[24] A. Bohr and B. R. Mottelson, Nuclear Structure (World Scientific Publishing, Singapore, 1998).
[25] E. J. Stephenson, J. C. Collins, C. C. Foster, D. L. Friesel, J. R. Hall, W. W. Jacobs, W. P. Jones, S. Kailas, M. Kaitchuck, P. Schwandt, W. W. Daehnick, and D. A. Goldberg, AIP Conf. Proc. 69, 484 (1981).

[26] A. Deltuva, Nucl. Phys. A 947, 173 (2016).

[27] A. Koning and J. Delaroche, Nucl. Phys. A 713, 231 (2003).

[28] R. Varner, W. Thompson, T. McAbee, E. Ludwig, and T. Clegg, Phys. Rep. 201, 57 (1991).

[29] R. Machleidt, Phys. Rev. C 63, 024001 (2001).

[30] R. De Leo, G. D'Erasmo, A. Pantaleo, M. N. Harakeh, S. Micheletti, and M. Pignanelli, Phys. Rev. C 23, 1355 (1981).

[31] I. J. Thompson and F. M. Nunes, Nuclear Reactions for Astrophysics (Cambridge University Press, Cambridge, 2009).

[32] S. Raman, C. W. Nestor Jr., and P. Tikkanen, At. Data Nucl. Data Tables 78, 1 (2001).

[33] T. Kibédi and R. Spear, At. Data Nucl. Data Tables 80, 35 (2002).

[34] S. Karataglidis, K. Amos, and B. G. Giraud, Phys. Rev. C 71, 064601 (2005).

[35] A. Diaz-Torres, I. J. Thompson, and C. Beck, Phys. Rev. C 68, 044607 (2003).

[36] L. C. Chamon, D. Pereira, M. S. Hussein, M. A. Cândido Ribeiro, and D. Galetti, Phys. Rev. Lett. 79, 5218 (1997).

[37] C. M. Perey and F. G. Perey, Phys. Rev. 132, 755 (1963).

[38] Y. Hirabayashi and Y. Sakuragi, Phys. Lett. B 258, 11 (1991).

[39] S. Santra, V. Parkar, K. Ramachandran, U. Pal, A. Shrivastava, B. Roy, B. Nayak, A. Chatterjee, R. Choudhury, and S. Kailas, Phys. Lett. B 677, 139 (2009).

[40] C. Beck, N. Keeley, and A. Diaz-Torres, Phys. Rev. C 75, 054605 (2007).

[41] S. Watanabe, T. Matsumoto, K. Minomo, K. Ogata, and M. Yahiro, Phys. Rev. C 86, 031601 (2012).

[42] J. Cook, Nucl. Phys. A 388, 153 (1982).

[43] E. Muskat, J. Carter, R. Fearick, and V. Hnizdo, Nucl. Phys. A 581, 42 (1995).

[44] R. Taylor, N. Fletcher, and R. Davis, Nucl. Phys. 65, 318 (1965).

[45] K. C. C. Pires, R. Lichtenthäler, A. Lépine-Szily, V. Guimarães, P. N. de Faria, A. Barioni, D. R. Mendes Junior, V. Morcelle, R. Pampa Condori, M. C. Morais et al., Phys. Rev. C 83, 064603 (2011).

[46] J. H. Dave and C. R. Gould, Phys. Rev. C 28, 2212 (1983).

[47] A. Bonaccorso and R. J. Charity, Phys. Rev. C 89, 024619 (2014). 